\title{
Electrophysiological Substrates in Papillary Muscle Arrhythmias - Implications for Catheter Ablation
}

Deep Chandh Raja, ${ }^{1}$ Vickram Vignesh Rangaswamy, ${ }^{2}$ Sreevilasam Pushpangadhan Abhilash, ${ }^{1}$ Kieran King ${ }^{3}$ and Rajeev K Pathak'

1. Canberra Hospital, Australian National University, Canberra, Australian Capital Territory, Australia; 2. Department of Cardiac Electrophysiology, Care Hospitals, Hyderabad, India; 3. Biosense Webster, Johnson \& Johnson Medical Pty Ltd, Canberra, Australian Capital Territory ", Australia

$\mathrm{P}$ apillary muscles are increasingly being recognised as a source of premature ventricular complexes (PVCS) and ventricular arrhythmias (VAs). These VAs, though amenable to catheter ablation, present unique challenges to an electrophysiologist. The endocavitary location, varied anatomy and anisotropic conduction of impulses within the papillary muscle are responsible for the characteristic electrophysiological observations - pleomorphic PVCs on electrocardiogram, abnormal potentials during mapping of PVCs and difficulties in ablation. Papillary muscles are also involved in malignant mitral valve prolapse syndromes, post-infarct VAs and triggers for PVC-induced ventricular fibrillation.

\section{Keywords}

Papillary muscle, ventricular tachycardia, catheter ablation

Disclosures: Deep Chandh Raja, Vickram Vignesh Rangaswamy, Sreevilasam Pushpangadhan Abhilash, Kieran King and Rajeev K Pathak have no financial or non-financial relationships or activities to declare in relation to this article. Acknowledgments: Dr Pathak is supported by an early career fellowship from the National Health and Medical Research Council of Australia.

Review Process: Double-blind peer review. Compliance with Ethics: This article involves a review of the literature and did not involve any studies with human or animal subjects performed by any of the authors. No information has been included that could reveal patient identity where representative electrocardiograms and imaging have been included. Authorship: The named authors meet the International Committee of Medical Journal Editors (ICMJE) criteria for authorship of this manuscript, take responsibility for the integrity of the work as a whole, and have given final approval for the version to be published.

Access: This article is freely accessible at touchCARDIO.com (c) Touch Medical Media 2020.

Received: 20 November 2019

Accepted: 16 December 2019

Published Online: 10 March 2020

Citation: European Journal of Arrhythmia \& Electrophysiology. 2020;6(1):32-8

Corresponding Author: Rajeev Kumar Pathak, Cardiac Electrophysiology Unit, Department of Cardiology, Canberra Hospital, Yamba Drive, Garran, ACT 2605, Australia

E: rajeev.pathak@act.gov.au; Twitter ID: @drrpathak

Support: No funding was received fo

the publication of this article.
Premature ventricular complexes (PVCS) can cause significant left ventricular (LV) dysfunction, leading to debilitating symptoms. ${ }^{1}$ Multiple single-centre studies have shown that catheter ablation is effective in curing these PVCs. ${ }^{2,3}$ However, catheter ablation of ventricular arrhythmias (VAs) originating from papillary muscles can be challenging. ${ }^{2}$ This is because of the heterogenous electrophysiological substrate that is characteristically observed in papillary muscle VAs. The varied anatomy of papillary muscles, different sites of origin and exits of VAs, role of underlying structural heart disease and concomitant mitral valve involvement can lead to diverse electrophysiological observations in papillary muscle VAs. In this article, we discuss the complex electrophysiological substrate in papillary muscle VAs, including anatomical features, electrocardiogram (ECG) characteristics, potential pathophysiological substrates, typical electrophysiological observations and characteristics of catheter ablation.

\section{Anatomical features of papillary muscles}

Location and architecture

Papillary muscles are endocavitary structures located in the mid to the apical third of the ventricular cavity. ${ }^{3}$ The challenges for catheter ablation, in addition to the endocavitary location, are that the anatomy of the papillary muscles is considerably variable in shape, number and attachments. The LV papillary muscles include the posteromedial and anterolateral groups. The posteromedial papillary muscle is located between the ventricular septum and posterior LV free wall. The anterolateral papillary muscle lies on the anterolateral LV free wall. ${ }^{4}$ The anterolateral papillary muscle is usually single, unlike multiple groups of the posteromedial papillary muscles. Each papillary muscle can have multiple heads and can be either 'tethered' or 'finger-like'. The tethered papillary muscles are attached to the LV free wall by means of numerous trabecular ridges. The finger-like papillary muscles are usually attached to the LV free wall over a broad base. The right ventricle (RV) papillary muscles include the anterolateral, posteromedial and septal muscle groups. LV posteromedial papillary muscles are a commoner source of papillary muscle VAs than those from LV anterolateral papillary muscles. ${ }^{5}$ Likewise, the RV septal papillary muscles are the commonest source of RV papillary muscles VAS. ${ }^{6}$

\section{Activation and preferential conduction within the papillary muscles}

The papillary muscles are embedded by a sub-endocardial layer of Purkinje network, which are more concentrated at the base and hence the papillary muscles get activated from the base to the tip. ${ }^{7.8}$ The site-of-origin of the papillary muscle VAs could be from the Purkinje-myocardial interface or deep within the myocardium anywhere along the length of the papillary muscle. These impulses eventually exit into the free walls of the ventricle. The architecture of the papillary muscles is complex, with multiple intervening myocardial strands resulting in anisotropic conduction of the depolarising stimuli. Preferential conduction along the length of these strands and variable exit into the ventricular walls is responsible for the various morphologies of QRS observed at the baseline as well as during an ablation. ${ }^{5}$ Additionally, false tendons could connect the papillary 
Figure 1: Twelve-lead electrocardiograms of posterior papillary muscle ventricular tachycardia and posterior fascicular ventricular tachycardia

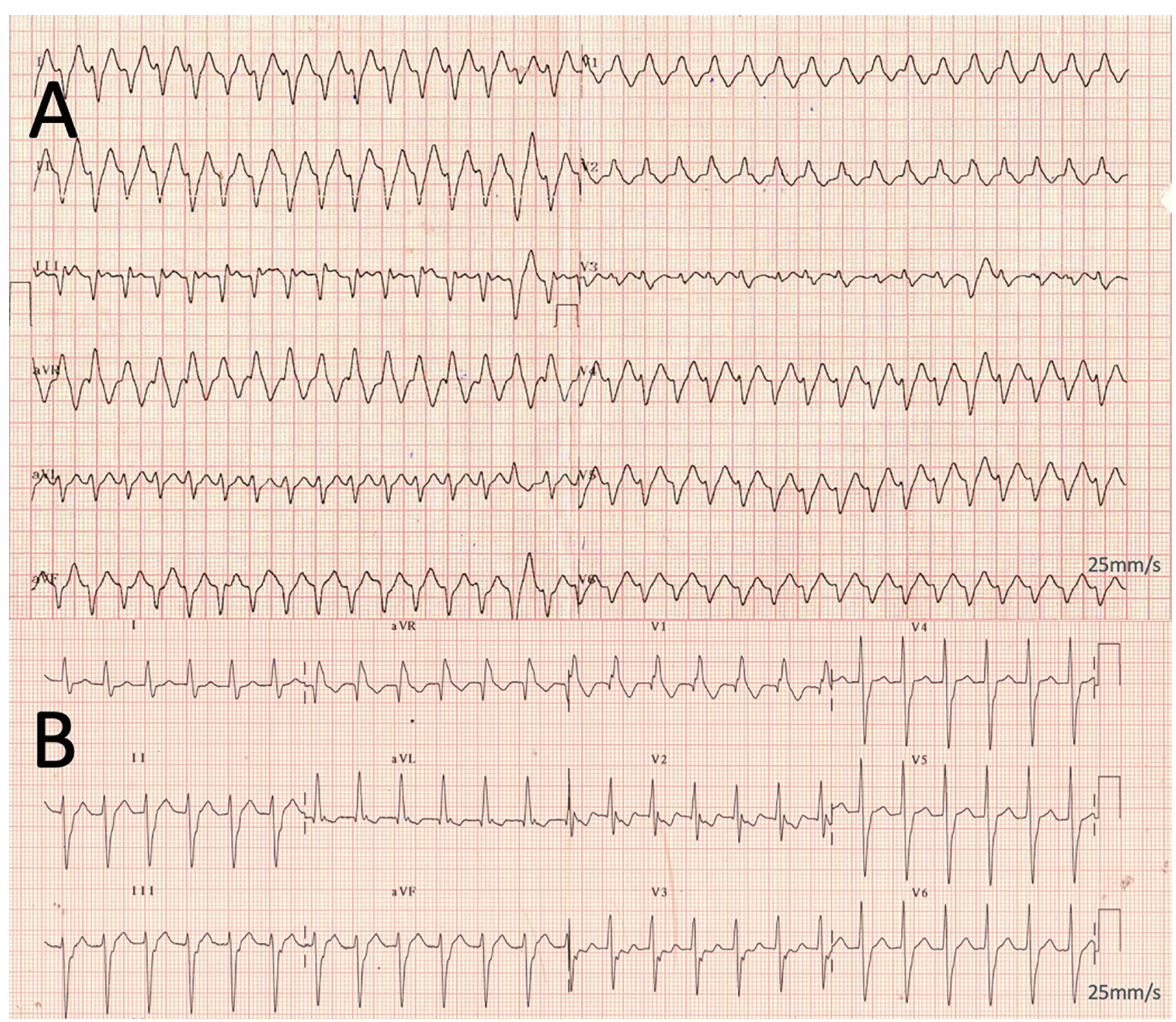

Panel A shows the 12-lead ECG of a patient who had VT arising from the poster-medial papillary muscle. Note the wide QRS (160 ms), monomorphic R in V1, extreme left axis deviation and the atypical RBBB pattern. This morphology of VT has to be distinguished from a left posterior fascicular VT which also has left axis deviation, as shown in another patient (Panel B). Note the relatively narrow QRS (120 ms), typical RBBB pattern ( $r S R^{\prime}$ in V2) and q waves in I, aVL.

$E C G$ = electrocardiogram; RBBB = right bundle branch block; $V T=$ ventricular tachycardia.

muscles to other regions of the ventricle and may serve as conduits for the conduction of impulses resulting in varied morphologies. ${ }^{9}$

\section{Electrocardiogram characteristics of papillary muscle ventricular arrhythmias \\ Hallmark electrocardiogram features}

LV papillary muscle VAs have close resemblance to fascicular VAs exiting around the same regions in the ventricle. Papillary muscle VAs characteristically lack the typical right bundle branch block (RBBB) pattern ( $\mathrm{rSR}^{\prime}$ pattern in $\mathrm{V} 1 / \mathrm{V} 2$ ) and more often bear a $\mathrm{qR}$ or a monomorphic R pattern in V1 - the atypical RBBB pattern. Also, the discrete q waves characteristic of fascicular VAS, may not be seen in the inferior or lateral leads. ${ }^{10,11}$ QRS notching in the PVCS is more often a feature of papillary muscle VAs than the fascicular VAs. The delay in conduction of the impulses from the papillary muscle into the surrounding myocardium could explain both the notching and the wideness of the QRS in papillary muscle VAs. The presence of VA QRS $\leq 130$ ms can differentiate fascicular VAs from papillary muscle VAs with 100\% sensitivity and specificity (Figures 1 and 2). RBBB pattern of PVCS with 'inferior limb discordance' (defined as predominant negative QRS in lead II and positive in lead III) may suggest exit from LV anterolateral papillary muscle. ${ }^{12}$ The mitral annular VAs originate from the more basal free wall of the LV. A positive precordial lead concordance favours mitral annular VA over papillary muscle VAS.

\section{Pleomorphic premature ventricular complexes}

Multiple morphologies of PVCs (pleomorphic PVCS) can be observed at the baseline (Figure 2). Presence of pleomorphic PVCs in a 12-lead ECG with atypical RBBB pattern should suggest possible origin from the LV papillary muscles. A number of mechanisms account for these multiple morphologies, such as varying origin within the papillary muscle, shifting exits due to preferential conduction and changing conduction delays due to the variable fiber orientation. ${ }^{13,14} \mathrm{~A}$ PVC burden $\geq 24 \%$ and presence of pleomorphic PVCs has been shown to be strongly associated with 


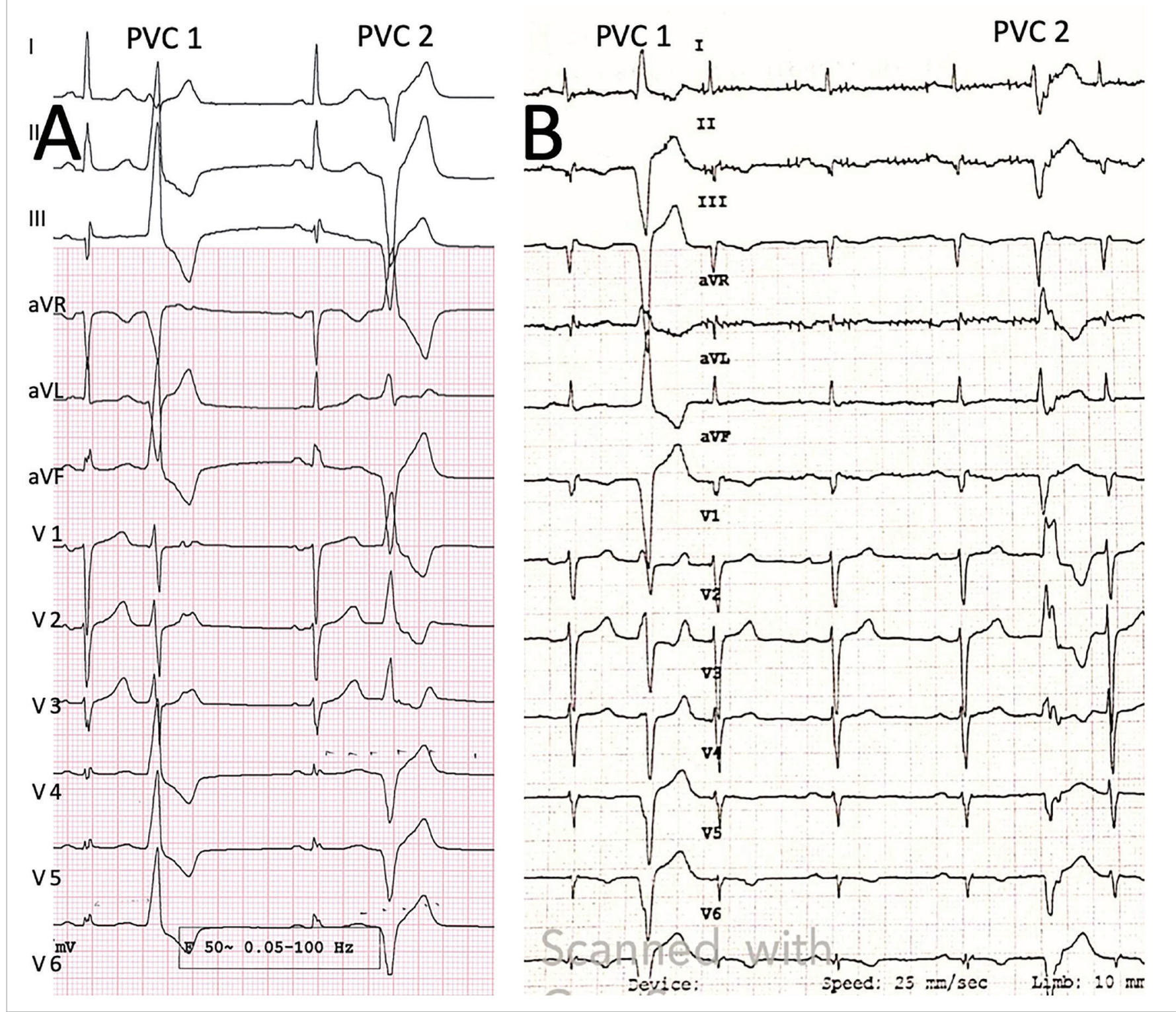

Note the different morphology of PVCS displayed in both panels A and B - pleomorphic PVCS. PVC 2 was the predominant PVC in both the patients - note the characteristic notching and wideness of the PVC, suggesting delayed conduction within the papillary muscle. PVC 1 in both patients is relatively narrow with atypical LBBB pattern suggesting LV septal exit. Both patients had documented LV 'false tendons' explaining the septal exit of the PVC from the posteromedial papillary muscle. $\angle B B B=$ left bundle branch block; $L V=$ left ventricular; $P V C=$ premature ventricular complex.

PVC-induced cardiomyopathy. ${ }^{15-7}$ Detection of pleomorphic PVCs from papillary muscles can also suggest poorer outcomes from ablation. ${ }^{18}$

\section{Potential pathophysiological substrates}

Precipitating factors

Papillary muscle VAs can occur in both structurally normal and diseased hearts. ${ }^{10}$ Papillary muscles are involved in a number of cardiac pathologies like ischaemia, infiltrative disorders and congenital malformations. They could be a part of a diffuse cardiomyopathy like in endocardial fibroelastosis, amyloidosis, sarcoidosis and ischaemic cardiomyopathy. ${ }^{19}$ Hypoxia, necrosis, fibrosis and calcification of the papillary muscles can be a potential source of malignant VAs. In patients with mitral valve prolapse (MVP) syndromes, a mechanical stretch-mediated injury to the papillary muscles is the likely trigger for automaticity observed with the papillary muscles. ${ }^{20} \mathrm{MVP}$ results in fibrosis of the papillary muscles in the long term, which can in turn serve as a substrate for VAs. ${ }^{21}$
Papillary muscle premature ventricular complex-triggered ventricular fibrillation Papillary muscles are under-recognised sites of triggers for PVC-induced ventricular fibrillation. A significant proportion of patients (nearly 25\%) with documented PVC-induced ventricular fibrillation have triggers arising from the papillary muscle. ${ }^{22}$ Abnormal automaticity within the Purkinje system, more commonly in the setting of ischaemic heart disease, can lead to exit into the Purkinje-papillary muscle interface leading to malignant papillary muscle VAs such as ventricular fibrillation/polymorphic ventricular tachycardia (VT). ${ }^{8}$

\section{Electrophysiological characteristics and implications}

Table 1 enlists studies and their pertinent findings with respect to electrophysiological characteristics of papillary muscle arrhythmias. $2,10,11,13-5,21-30$ 
Table 1: Studies on papillary muscle arrhythmias and their characteristics

\begin{tabular}{|c|c|}
\hline Authors (year) & Characteristic features \\
\hline \multicolumn{2}{|l|}{ Electrocardiography features } \\
\hline Yamada et al. $(2010)^{11}$ & Pleomorphic PVCs \\
\hline Al'Aref et al. (2015) ${ }^{15}$ & $\begin{array}{l}\text { Algorithm for differentiating papillary muscle } \\
\text { from mitral annular and fascicular arrhythmias }\end{array}$ \\
\hline \multicolumn{2}{|c|}{ Electrophysiological properties } \\
\hline Doppalapudi et al. (2008)26 & Activation time \\
\hline $\begin{array}{l}\text { Good et al. }(2008)^{10} \\
\text { Yamada et al. }(2010)^{11} \\
\text { Ban et al. }(2013)^{24}\end{array}$ & Pre-potentials \\
\hline Ban et al. $(2013)^{24}$ & Unipolar EGM characteristics \\
\hline Itoh et al. $(2017)^{14}$ & Preferential conduction and pace mapping \\
\hline Koutbi et al. $(2018)^{27}$ & High-density mapping \\
\hline Komatsu et al. $(2017)^{25}$ & Fascicular-papillary muscle VT \\
\hline Enriquez et al. (2019) ${ }^{21}$ & Association with mitral valve prolapse syndromes \\
\hline Van Herendael et al. (2014) $)^{22}$ & PVC-triggered ventricular fibrillation \\
\hline Bogun et al. $(2008)^{23}$ & Post-infarction papillary muscle arrhythmias \\
\hline \multicolumn{2}{|c|}{ Outcomes of catheter ablation } \\
\hline Yokokawa et al. $(2010)^{13}$ & Procedure time \\
\hline $\begin{array}{l}\text { Latchamsetty et al. (2015)² } \\
\text { Yokokawa et al. (2010) }\end{array}$ & Outcomes of ablation \\
\hline $\begin{array}{l}\text { Rivera et al. }(2016)^{28} \\
\text { Gordon et al. }(2018)^{29}\end{array}$ & Cryoablation \\
\hline \multicolumn{2}{|l|}{ Role of imaging } \\
\hline Kautzner et al. (2019) 30 & Role of intra-cardiac echocardiography \\
\hline Yokokawa et al. $(2010)^{13}$ & Papillary muscle size and lesion volume \\
\hline
\end{tabular}

$E G M=$ electrogram; $P V C=$ premature ventricular complex;

$V T=$ ventricular tachycardia.

\section{Induction and mechanisms of papillary muscle ventricular arrhythmias}

Both automatic and re-entry mechanisms of arrhythmogenesis have been postulated in papillary muscle VAs depending on the substrate. ${ }^{3}$ The usual presentation of papillary muscle VAs due to automaticity is in the form of frequent PVCs or non-sustained VT. Presentation with sustained $\mathrm{VT}$ is possible, and more so in the background of exertion and more often points to a re-entry circuit. A re-entry mechanism is more likely when there is heterogeneity in the refractoriness of the papillary muscle due to an underlying structural heart disease like a prior myocardial infarction. ${ }^{233.31}$ Also, a 'source-sink hypothesis' could be attributed to the triggering of VAs in the presence of a substrate within the papillary muscle - the depolarising current from the Purkinje network can unload into the papillary muscles and trigger an arrhythmia. ${ }^{7.24}$ Papillary muscle VAs arising in the zone of a prior myocardial infarction may also be sites of critical isthmus of the re-entry circuit of the VT.12 The Purkinje network surrounding the papillary muscles may serve as regions of slow conduction of a fascicular VT (FVT). This represents a distinct subtype of verapamil-sensitive FVTs. ${ }^{25}$ Not only do the 12-lead ECG characteristics of papillary muscle FVT resemble the usual FVTs, but also these re-entry circuits are sensitive to verapamil (prolong or terminate). FVTs can be reproducibly induced with programmed atrial/ventricular stimulation. ${ }^{25}$

As most of the papillary muscle VAs are catecholamine-sensitive, they can be induced with isoproterenol/epinephrine. In the presence of a substrate within the papillary muscle, where the mechanism is likely to be re-entry, the VAs can be induced with programmed atrial or ventricular pacing. Non-inducibility with atrial or ventricular pacing and suppression of the VA upon overdrive pacing without satisfying any criteria for entrainment suggest automaticity as the likely mechanism of papillary muscle VAs and is usually noted in structurally normal hearts. ${ }^{26}$

\section{Mapping of abnormal potentials}

Mapping for the earliest activation signals is the cornerstone for successful ablation of the papillary muscle VAs. Earliest activation signals of $34 \pm 15 \mathrm{~ms}$ have shown to be effective for successful ablations. ${ }^{10}$ Recognition of 'pre-potentials' is an essential part of activation mapping. There have been frequent observations in the past that pre-potentials could be noted at the sites of successful ablation of papillary muscle VAs. These pre-potentials may be seen in $30-56 \%$ cases, ${ }^{10,26,31}$ and if noted, could predict successful outcomes of ablation (Figure 3). ${ }^{13}$ The genesis of these pre-potentials has been much debated. There have been three types of pre-potentials described: 1. high-frequency (sharp) potentials; 2. high amplitude low-frequency (rounded) potentials; and 3. Iow amplitude low-frequency (rounded) potentials. The high-frequency potentials are the Purkinje potentials and, when also found during the sinus rhythm, suggest a superficial origin of the VAS from the Purkinje-myocardial interface. These Purkinje potentials could be observed in up to $45 \%$ cases. ${ }^{10}$ When separated from the ventricular potential by an iso-electric line, these Purkinje potentials may suggest a delayed exit into the myocardium due to slow conduction. ${ }^{32}$ The low-frequency potentials are likely to be of myocardial origin (myo-potentials). These myo-potentials should suggest deeper origin of the VAs from the papillary muscle. The amplitude of these potentials should be able to suggest near-field (high amplitude) and the far-field (low amplitude). The far-field potentials suggest deep intra-mural origin or an epicardial exit of the VAs.

Detection of abnormal potentials like late systolic or early/late diastolic potentials at sites of early activation during sinus rhythm or during PVCS should suggest an abnormal substrate. The diastolic potentials found on the papillary muscles may serve as targets for ablation in patients with FVT. Mapping with multi-electrode catheters has not been much studied and it remains to be seen if they have better sensitivity than mapping with conventional quadripolar catheters in picking up the pre-potentials. ${ }^{27}$ Unipolar mapping is important to understand and differentiate the near-field and far-field early potentials. ${ }^{33}$ Presence of $\mathrm{Q}$ waves coinciding with the onset of the early pre-potentials should suggest the subjacent source of the VAs to the mapping catheter. The slow downstroke of $>50 \mathrm{~ms}$ of the $\mathrm{Q}$ wave of the unipolar electrogram has been shown to predict success; however, this was studied only in a small cohort of patients. ${ }^{24}$

\section{Pace mapping at the papillary muscles}

Pace mapping at exit sites may not be representative of the site of origin. During pace mapping, the morphology of the QRS on the 12-lead ECG can vary depending on the pacing output, the amount of myocardium captured, and underlying scar, if any. Pace mapping should be at an output just above threshold to ensure capture of only the local myocardium. The 12-lead pace map should be interpreted with caution keeping in mind the property of preferential conduction of the papillary muscle VAs. ${ }^{13,26} \mathrm{~A}$ good pace map, when associated with early activation signals, can define success in nearly $70 \%$ cases

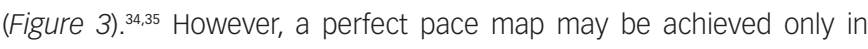
30-60\% cases. ${ }^{10,34}$ Nevertheless, pace mapping can be a strategy for papillary muscle VA ablation and is especially useful when there are no inducible VAs or in haemodynamically unstable VAs precluding an 
Figure 3: Electrophysiolgical characteristics of mapping and ablation in a patient with posteromedial papillary muscle ventricular arrhythmia

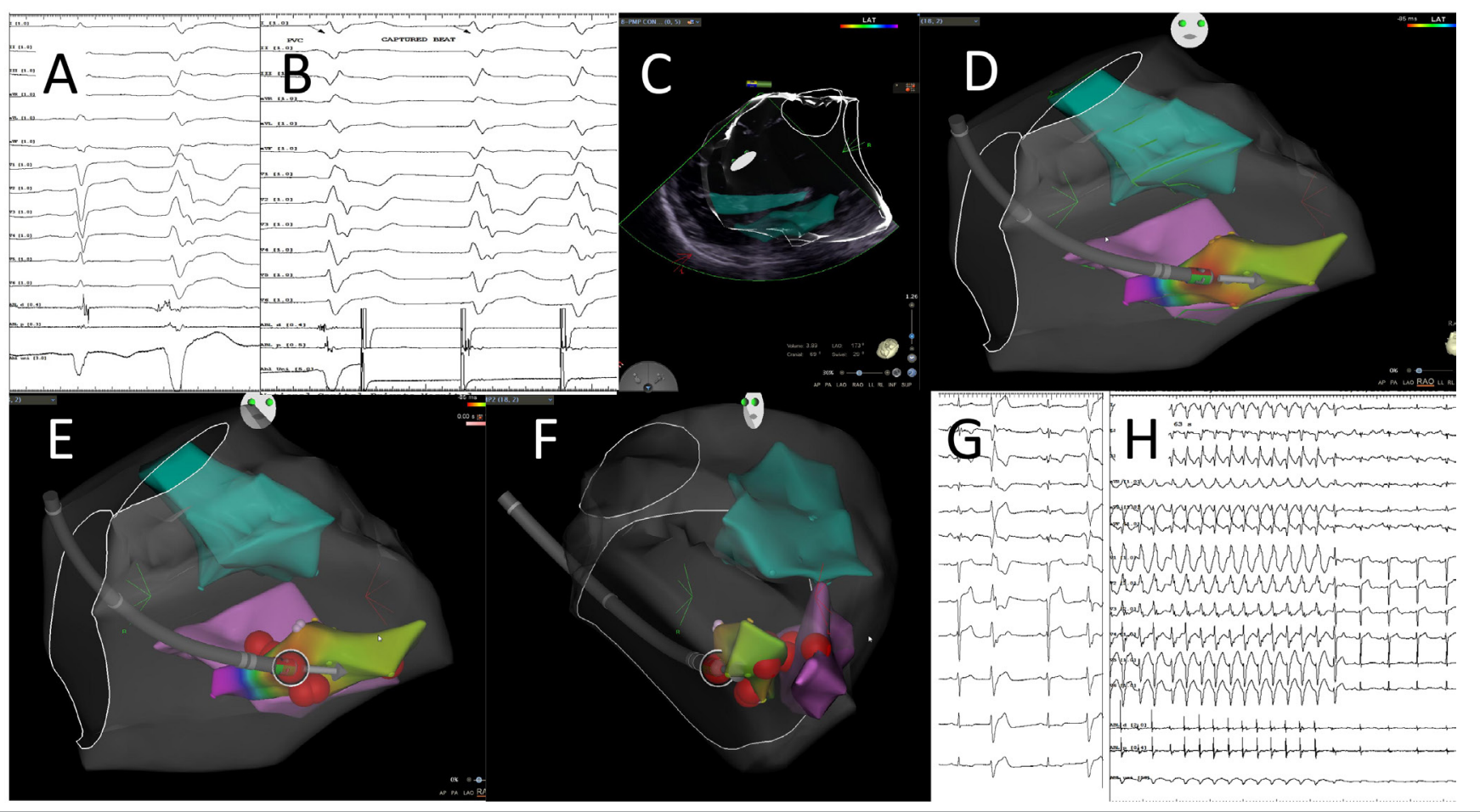

Panel A shows the early activation signals at the septal head of the papillary muscle - note the reversal of activation of the pre-potentials during the PVC and in sinus rhythm Pace map at the same site (Panel B) resulted in near perfect 12/12 morphology match and stim-QRS latency of 36 ms. Panel C shows integration of ICE imaging to delineate the posteromedial papillary muscle group. Panels D shows the 3D electroanatomical maps in LAO view (anterolateral papillary muscle - teal colour; posteromedial papillary muscle - pink colour). The mid region of the medial aspect of the septal head showed the earliest activation signals (LAT colour map). Panels E and F, in RAO and LAO views respectively, show the ablation lesions as red spheres - note the intentional lateral force as seen by the force vector at the tip of the contact force catheter. Panel $G$ shows change in morphology in the PVC after the start of ablation. Panel H shows an acceleration of the VT before successful termination.

$E C G=$ electrocardiogram; ICE = intra-cardiac echocardiography; $L A O=$ left anterior oblique; $L A T=$ local activation time; $L V=$ left ventricular; $P V C=$ premature ventricular complex; $R A O=$ right anterior oblique; $V T=$ ventricular tachycardia.

activation map and when ablation at earliest activation sites fail. Good pace maps can be obtained at multiple sites remote to the earliest activation sites and ablation at these sites might be successful when VAs are not inducible. ${ }^{35}$

Understanding of the stimulus-QRS duration (latency) of the paced complex is important to understand the preferential conduction observed in papillary muscle VAs (Figures 3 and 4). It is possible that the paced QRS matches the morphology of the PVC, yet the site of pacing might be distal to the site of origin. Therefore, pace mapping of the papillary muscle VAs should consider not only the morphology match but also the latency of capture as it has been shown that ablation at these sites is successful when previous attempts have failed at the earliest

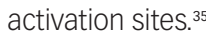

\section{Charateristics of catheter ablation in papillary muscle ventricular arrhythmias} Short- and long-term success rates

The acute success rate of ablation varies from $60-100 \%$, but the long-term success rate of these arrhythmias was found to be less promising $(60 \%)$, as shown in a large multicentre study. ${ }^{2}$ The recurrence rates with respect to anterolateral and posteromedial papillary muscles has been noted to be $71 \%$ and $50 \%$, respectively. ${ }^{34,36}$ The higher recurrence rates observed in the ablation of papillary muscle VAs are because the papillary muscles are very thick structures and arrhythmic foci of papillary muscle VAs are more often deeper from the endocardium. The depth of the radiofrequency lesion may not encompass the deep focus. Also, the close proximity of the heads of the papillary muscles and their vigorous contraction during systole can pose difficulties during catheter manipulation due to unstable catheter positions. Considering the deeper origin of the papillary muscle VAs, the choice of catheter is always an irrigation catheter to create a deeper and wider lesion. Ablation with conventional non-irrigation catheters has, in fact, been shown to have recurrences. ${ }^{26}$ Establishing contact with an endocavitary structure like the papillary muscle has been recognised as the most challenging aspect of the ablation. Therefore, use of contact-force catheters and adjunct imaging guidance like intra-cardiac echocardiography (ICE) can aid in improving success rates. ${ }^{37}$

Cryoablation is now established as an alternate modality in papillary muscle VA ablation especially when catheter contact is an issue or in cases with recurrences with radiofrequency ablation (RFA). ${ }^{28}$ The advantage of cryoablation is the catheter-tissue adherence at extremely low temperatures increasing likelihood of a stable catheter position. The cryoablation catheter can also target multiple different surfaces of the papillary muscle. Acute success has been reported to be $100 \%{ }^{36}$ Excellent success rates (93.8\%) have also been reported in cases of failed papillary muscle VA ablation with RFA. ${ }^{29}$ The disadvantages of cryoablation are reduced manoeuverability due to bulky catheters, reduced lesion depth and inability to accurately project the catheter tip onto the electroanatomic map.

\section{Sites of ablation}

RFAs are usually carried out at the base of the papillary muscle, as ablating the head of the papillary muscle can potentially risk in damage 
Figure 4: Electrophysiolgical characteristics of mapping and ablation in a patient with anterolateral papillary muscle ventricular arrhythmias
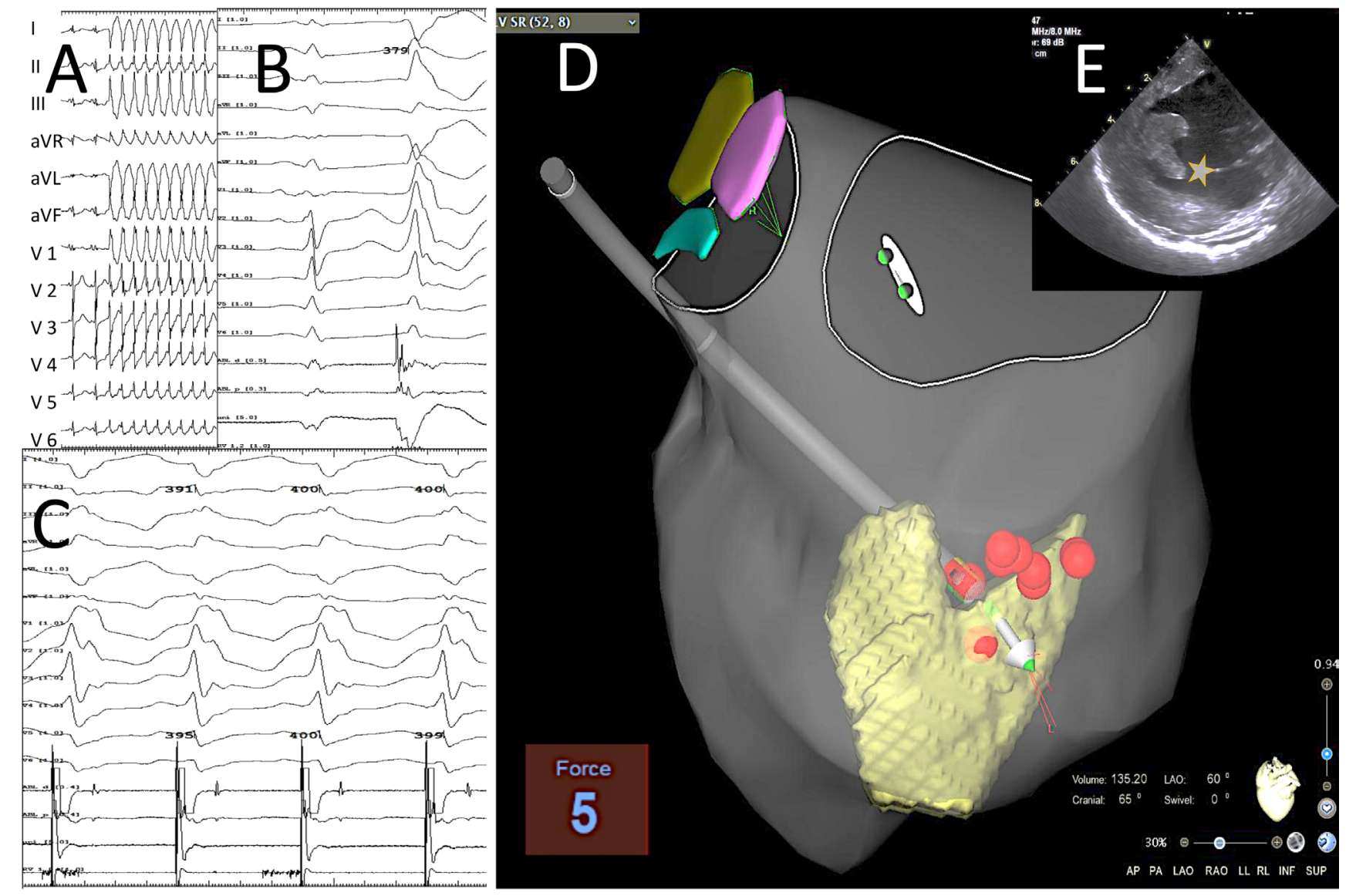

Panel A shows the 12-lead ECG of a patient with anterolateral papillary muscle PVCs. Panel B shows the pre-potentials (38 ms), bipolar and unipolar signals at the lateral head of the papillary muscle. Pace map at this site (Panel C) showed a near perfect 12/12 morphology match and stim-QRS latency of 38 ms. Panel D shows the 3D electroanatomical map in the LAO cranial view. LV geometry, integrated using ICE imaging (background grey); septal and lateral head of the papillary muscle (yellow colour); ablation lesions (red spheres); force vector (arrow with green tip on the contact force catheter). Panel E shows ICE image - note the two heads of the 'bifid' anterolateral papillary muscle with hyper-echogenicity at the tip of the lateral head (star).

$E C G$ = electrocardiogram; ICE = intra-cardiac echocardiography; $L A O=$ left anterior oblique; $L V=$ left ventricular; $R B B B=$ right bundle branch block; $P V C S=$ premature ventricular complexes; $V T=$ ventricular tachycardia.

to the chordae resulting in mitral valve dysfunction. 'Circumferential' ablation at the base of the papillar muscle can isolate the muscle and block all exits into the LV. Such a strategy has been shown to be effective in reducing recurrences. ${ }^{31}$ The number of RF lesions required for elimination of papillary muscle VAs is significantly higher than those for fascicular VAs. ${ }^{10,11}$ This is more related to the complex anatomy, difficulties in maintaining contact and deeper foci within the papillary muscles. The retrograde aortic approach to both the LV papillary muscles is helpful in most cases. Trans-septal access can be attained for better access to the postero-medial group of papillary muscles or the lateral heads of the anterolateral papillary muscles.

\section{Role of multi-modality imaging}

ICE imaging during ablation of papillary muscle PVCs enables visualisation of the head, body and base of the papillary muscles. More importantly ICE helps in establishing contact of the ablation catheter during RFA. ICE has been extensively used in ablation and has become the standard-of-care for ablation of VAs from endocavitary structures like the papillary muscles. ${ }^{30}$ Moreover, real-time integration of ICE imaging with 3D electro-anatomical mapping enables precise delineation of the geometry of the papillary muscles in relation to the cavity (Figures 3 and 4). ${ }^{37}$ ICE can also identify areas of high echogenicity which may correspond to areas of low bipolar voltage and scar. ${ }^{21,38}$ Ablation at the parietal region of the RV infundibular muscles have high recurrence rates and is technically challenging due to the location and ICE can play a crucial role in establishing contact with these structures. ${ }^{39}$ The 3D electro-anatomical mapping systems have advanced to enable integration of ICE, computed tomography (CT) and magnetic resonance imaging (MRI) to aid precise mapping of papillary muscles. Additionally, advanced tools such as automated pace mapping modules and integration of ICE, CT and MRI algorithms within the 3D electro-anatomical mapping systems can help in achieving better success rates during ablation of these complex structures in both the $\mathrm{LV}$ and the RV.,40,41 $\mathrm{A}$ larger arrhythmogenic mass of the papillary muscles detected on cardiac MRI can also correlate with poorer outcomes from RFA. ${ }^{13}$

\section{Observations during ablation}

Morphology change can be observed during ablation of papillary muscle VAs in 47\% cases. ${ }^{34}$ A morphology change observed during or after delivery of RFA burns suggests a shift in the preferential conduction along the length of the papillary muscle and exit of the PVC from a different site at the LV free wall, though the source continues to remain the same (Figure 3). The morphology of the newer PVC should 
be studied, and activation mapping has to be repeated for the same. More often, this may necessitate ablation on both sides of the papillary muscle to get rid of the PVCs. ${ }^{5,34}$ In patients with FVTs, a change in the 12-lead morphology of the tachycardia upon ablation at the routine target sites of ablation such as the posterior/anterior fascicular sites, along with a reproducible intiation of the tachycardia, should alert the operator to look for diastolic potentials in the papillary muscles as these structures are known to be involved in the circuits of FVTs. Successful ablation of these abnormal potentials may eliminate these tachycardias. ${ }^{25}$ Acceleration of PVCS or induction of VT is another frequently observed characteristic during the ablation of papillary muscle VAs.

\section{Conclusion}

The electrophysiological substrate noted in papillary muscles is unique and presents challenges during ablation. Awareness of the anatomical variations, correlation of the ECG characteristics with electrophysiological observations, an in-depth understanding of the activation signals during mapping of the PVCs and integration of multi-imaging modalities during ablation help in achieving reasonable success in ablation of papillary muscle VAs. It is also important to recognise distinct clinical entitites associated with papillary muscle VAs like the MVP syndromes, PVC-triggered malignant VAs and ischaemia-driven papillary muscle VAs. $\square$
1. Pathak RK, Ariyarathna N, Garcia FC, et al. Catheter ablation of idiopathic ventricular arrhythmias. Heart Lung Circ. 2019;28:102-9.

2. Latchamsetty R, Yokokawa M, Morady F, et al. Multicenter outcomes for catheter ablation of idiopathic premature ventricular complexes. JACC Clin Electrophysiol. 2015:1:116-23.

3. Enriquez A, Supple GE, Marchlinski FE, Garcia FC. How to map and ablate papillary muscle ventricular arrhythmias. Heart Rhythm. 2017:14:1721-8.

4. Naksuk N Kapa S, Asirvatham SJ. Spectrum of ventricular arrhythmias arising from papillary muscle in the structurally normal heart. Card Electrophysiol Clin. 2016;8:555-65.

5. Yamada T, Kay GN. Anatomical consideration in catheter ablation of idiopathic ventricular arrhythmias. Arrhythm Electrophysiol Rev. 2016;5:203-9.

6. Santoro F, L DIB, Hranitzky P, et al. Ventricular tachycardia originating from the septal papillary muscle of the right ventricle: electrocardiographic and electro-physiologica characteristics. J Cardiovasc Electrophysiol. 2015;26:145-50.

7. Rawling DA, Joyner RW, Overholt ED. Variations in the functional electrical coupling between the subendocardial Purkinje and ventricular layers of the canine left ventricle. Circ Res. 1985;57:252-61.

8. Syed FF, Ackerman MJ, McLeod CJ, et al. Sites of successful ventricular fibrillation ablation in bileaflet mitral valve prolapse syndrome. Circ Arrhythm Electrophysiol. 2016:9 pii:e004005.

9. Syed FF, Hai JJ, Lachman N, et al. The infrahisian conduction system and endocavitary cardiac structures: relevance for the invasive electrophysiologist. I Interv Card Electrophysio. 2014;39:45-56.

10. Good E, Desjardins B, Jongnarangsin K, et al. Ventricular arrhythmias originating from a papillary muscle in patients without prior infarction: a comparison with fascicular arrhythmias. Heart Rhythm. 2008;5:1530-7.

11. Yamada T, Doppalapudi $H$, McElderry $H T$, et al. Electrocardiographic and electro-physiological characteristics in idiopathic ventricular arrhythmias originating from the papillary muscles in the artich left ventricle: relevance for cat

12. Enriquez A, Pathak RK, Santangeli P, et al. Inferior lead discordance in ventricular arrhythmias: A specific marker for certain arrhythmia locations. J Cardiovasc Electrophysiol. 2017;28:1179-86.

13. Yokokawa M, Good E, Desjardins B, et al. Predictors of successful catheter ablation of ventricular arrhythmias arising from the papillary muscles. Heart Rhythm. 2010;7:1654-9.

14. Itoh T, Yamada T. Preferential conduction during posterior papillary muscle origin premature ventricular contractions demonstrated by pace mapping. J Cardiovasc Electrophysio 2017;28:235-6.

15. Al'Aref SJ, ID JE, Markowitz SM, et al. Differentiation of papillary muscle from fascicular and mitral annular ventricular arrhythmias in patients with and without structural heart disease. Circ Arrhythm Electrophysiol. 2015;8:616-24.

16. Baman TS, Lange $\mathrm{DC}$, llg KJ, et al. Relationship between burden of premature ventricular complexes and left ventricular function. Heart Rhythm. 2010;7:865-9.

17. Sheldon SH, Latchamsetty R, Morady F, Bogun F. Catheter ablation in patients with pleomorphic, idiopathic, premature ventricular complexes. Heart Rhythm. 2017;14:1623-8.

18. Blaye-Felice MS Hamon D, Sacher $F$ et al Premature ventricular contraction-induced cardiomyopathy: related clinical and electrophysiologic parameters. Heart Rhythm. 2016;13:103-10.

19. Roberts WC, Cohen LS. Left ventricular papillary muscles. Description of the normal and a survey of conditions causing them to be abnormal. Circulation. 1972;46:138-54.

20. Markowitz SM. Mitral valve prolapse causes arrhythmias from the papillary muscles: A stretch of the truth or reality? Pacing Clin Electrophysiol. 2018;41:759-61.

21. Enriquez A, Shirai Y, Huang J, et al. Papillary muscle ventricular arrhythmias in patients with arrhythmic mitral valve prolapse: electrophysiologic substrate and catheter ablation outcomes J Cardiovasc Electrophysiol. 2019;30:827-35.

22. Van Herendael $\mathrm{H}$, Zado ES, Haqqani $\mathrm{H}$, et al. Catheter ablation of ventricular fibrillation: importance of left ventricular of ventricular fibrillation: importance of left ventricular
outflow tract and papillary muscle triggers. Heart Rhythm. 2014:11:566-73.

23. Bogun F, Desjardins B, Crawford T, et al. Post-infarction ventricular arrhythmias originating in papillary muscles. J Am Coll Cardiol. 2008;51:1794-802.

24. Ban JE, Lee HS, Lee DI, et al. Electro-physiological characteristics related to outcome after catheter ablation of idiopathic ventricular arrhythmia originating from the papillary muscle in the left ventricle. Korean Circ J. 2013;43:811-8.

25. Komatsu Y, Nogami A, Kurosaki K, et al. Fascicular ventricular tachycardia originating from papillary muscles: Purkinje network involvement in the reentrant circuit. Circ Arrhythm Electrophysiol. 2017;10 pii:e004549.

26. Doppalapudi $\mathrm{H}$, Yamada T, MCElderry HT, et al. Ventricular tachycardia originating from the posterior papillary muscle in the left ventricle: a distinct clinical syndrome. Circ Arrhythm the left ventricle: a distinct
Electrophysiol. 2008:1:23-9.

27. Koutbi L, Maille B, Peyrol M, et al. High-density mapping for catheter ablation of premature ventricular complexes originating from left ventricular papillary muscles: a case series Pacing Clin Electrophysiol. 2018;41:1071-7.

28. Rivera S, Ricapito Mde L, Tomas $L$, et al. Results of cryoenergy and radiofrequency-based catheter ablation for treating ventricular arrhythmias arising from the papillary muscles of the left ventricle, guided by intracardiac echocardiography and image integration. Circ Arrhythm Electrophysiol. 2016;9:e003874.

29. Gordon JP, Liang Jل, Pathak RK, et al. Percutaneous cryoablation for papillary muscle ventricular arrhythmias after failed radiofrequency catheter ablation. J Cardiovasc Electrophysio. 2018;29:1654-63

30. Kautzner J, Peichl P. Papillary muscle ventricular tachycardia or ectopy: diagnostics, catheter ablation and the role of intracardiac echocardiography. Arrhythm Electrophysiol Rev. 2019:8:65-9.

31. Wo HT, Liao FC, Chang PC, et al. Circumferential ablation at the base of the left ventricular papillary muscles: A highly effective approach for ventricular arrhythmias originating from the papillary muscles. Int J Cardiol. 2016;220:876-82.

32. Madhavan M, Asirvatham SJ. The fourth dimension: endocavitary ventricular tachycardia. Circ Arrhythm endocavitary ventricular tachycar

33. Liu XK, Barrett R, Packer DL, Asirvatham SJ. Successful management of recurrent ventricular tachycardia by electrical isolation of anterolateral papillary muscle. Heart Rhythm. 2008;5:479-82

34. Yamada T, Doppalapudi H, McElderry HT, et al. Idiopathic ventricular arrhythmias originating from the papillary muscles in the left ventricle: prevalence, electrocardiographic and electro-physiological characteristics, and results of the radiofrequency catheter ablation. J Cardiovasc Electrophysiol. 2010;21:62-9.

35. Itoh T, Yamada T. Usefulness of pace mapping in catheter ablation of left ventricular papillary muscle ventricular arrhythmias with a preferential conduction. J Cardiovasc arrhythmias with a preferential

36. Rivera S, Tomas L, Ricapito MP, et al. Updated results on catheter ablation of ventricular arrhythmias arising from the papillary muscles of the left ventricle. J Arrhythm. 2019;35:99-108

37. Yamada T, McElderry HT, Doppalapudi H, Kay GN. Real-time integration of intracardiac echocardiography and electroanatomic mapping in PVCs arising from the LV anterior papillary muscle. Pacing Clin Electrophysiol. 2009;32:1240-3.

38. Lee A, Hamilton-Craig C, Denman R, Haqqani HM. Catheter ablation of papillary muscle arrhythmias: Implications of mitral valve prolapse and systolic dysfunction. Pacing Clin mitral valve prolapse and systo

39. Yamada T, Yoshida N, Litovsky SH, et al. Idiopathic ventricular arrhythmias originating from the infundibular muscles: prevalence, electrocardiographic and electro-physiologica characteristics, and outcome of catheter ablation Circ Arrhythm Electrophysiol. 2018;11:e005749.

40. Nakahara S, Toratani N, Takayanagi K. Catheter ablation of ventricular tachycardia originating from the left posterior papillary muscle guided by the shadow of a multipolar catheter. Indian Pacing Electrophysiol J. 2012;12:186-9.

41. Chang YT, Lin YJ, Chung FP, et al. Ablation of ventricular arrhythmia originating at the papillary muscle using an automatic pacemapping module. Heart Rhythm. 2016:13:1431-40 